\title{
New Method of Solution for Unretarded Satellite Orbits ${ }^{1}$
}

\author{
John P. Vinti
}

(June 16, 1959)

\begin{abstract}
An axially symmetric solution of Laplace's equation in oblate spheroidal coordinates is found, which may be used as the gravitational potential about an oblate planet. This potential, which makes the Hamilton-Jacobi equation for a satellite orbit separable, has an expansion in zonal harmonics in which the amplitudes of the zeroth and second harmonics can be adjusted to agree exactly with the values for any axially symmetric planet and a fourth harmonic which then agrees approximately with the latest value for that of the earth. The net result is therefore a reduction of the problem of satellite motion to quadratures, with use of a potential field that is much closer to the empirically accepted one for the earth than any heretofore used as the starting point of a calculation. It may thus be possible to do the gravitational theory of a satellite orbit very accurately without use of perturbation theory. The method can take into account a first harmonic in the potential, in case observations are reduced to a center which does not coincide with the center of mass of the planet.
\end{abstract}

\section{Introduction}

The purpose of this paper is to develop a method of solution of the central problem of satellite astronomy, viz, the theory of the effect of the oblateness of a planet on the orbit of any satellite, bound or unbound, but near enough to the planet so that the forces of other bodies may be neglected. (A preliminary note has appeared in [1]. ${ }^{2}$ )

To do so, let us consider the gravitational potential $V$ outside an axially symmetric oblate planet of mass $M$. We call the axis of symmetry the polar axis; this will ordinarily be indistinguishable from the axis of rotation, except for entirely negligible periodic effects. We also choose some point $O$ on the polar axis as the "center" or origin of a coordinate system and let $\mathbf{r}$ be the corresponding position vector of a field point. With $r \equiv|\mathbf{r}|$, the declination $\theta$ as the complement of the angle between $\mathbf{r}$ and the polar axis, $G$ the gravitational constant, $M$ the mass of the earth, and $\mu \equiv G M$, the potential $V(r, \theta)$ can be expressed by means of an expansion in spherical harmonics:

$$
V=-\mu r^{-1}\left[1-\sum_{n=1}^{\infty} J_{n}(R / r)^{n} P_{n}(\sin \theta)\right] .
$$

Here $R$ is the equatorial radius, i.e., the radius of a section through $O$ perpendicular to the polar axis. The notation $J_{n}$ for the coefficients was introduced by Merson and King-Hele [2]. For points on the polar axis $(\theta=\pi / 2)$ at the distance $R$ from the center, the ratio of any two terms in (1) is equal to the ratio of the corresponding $J$ 's.

If the planet has symmetry with respect to the equatorial plane, all the odd harmonies drop out; i.e., $J_{1}=J_{3}=J_{5}=\ldots=0$. (In any case, even if no such symmetry exists, the first harmonic drops out, i.e., $J_{1}$ vanishes, if $O$ is chosen at the center of mass.) The coefficients $J_{2}$ and $J_{4}$ are given by

$$
\begin{gathered}
J_{2}=2 J / 3=\left(I_{p}-I_{t}\right) / M R^{2}, \\
J_{4}=-8 D / 35,
\end{gathered}
$$

where the quantities $J$ and $D$ were introduced by Jeffreys [3], and where $I_{p}$ and $I_{t}$ are the

1 This work was supported by the U.S. Air Force, through the Office of Scientific Research of the Air Research and Development Command.

2 Figures in brackets indicate the literature references at the end of this paper. 
moments of inertia of the planet about polar and transverse axes through $O$. For the earth, with $O$ taken at the center of mass, Jeffreys [3] gives the values

$$
J=(1637.5) 10^{-6} \quad D=(10.7) 10^{-6},
$$

while King-Hele and Merson [4] derive from recent satellite observations the values

$$
J=(1624.6 \pm 0.3) 10^{-6}, \quad D=(6 \pm 1) 10^{-6}, \quad J_{6}=(0.1 \pm 1.5) 10^{-6} .
$$

It should be noted that until very recently it has always been assumed that the earth has symmetry with respect to its equatorial plane - strictly speaking, that the geoid, or smoothed earth, has such symmetry. That is, all the odd $J$ 's have been assumed to vanish. Recently, however, there has appeared some evidence [5] for an observable asymmetry with respect to the equatorial plane. Such evidence, if confirmed, would have the following results. If $O$ is taken at the center of mass $C$, then $J_{1}$ would still vanish, but $J_{3}, J_{5}$. . . would have small nonvanishing values. If, on the other hand, $O$ is taken, either by error or by design, as noncoincident with $C$, but still on the polar axis, then we should have $J_{1} \neq 0$, with $J_{3}, J_{5}, \ldots$. still small but nonvanishing. The only conceivable difficulty that might arise with this latter choice for the center has to do with the inertial forces acting on the satellite. This difficulty is discussed and removed in the next section. A reason for deliberately choosing $O$ as noncoincident with $C$ might be to represent any equatorial asymmetry largely by means of a first harmonic, and such a procedure might work if the third and higher odd harmonics then turn out to be negligible in comparison with the first.

In the present paper we first derive a solution $V_{L}$ of Laplace's equation, in oblate spheroidal coordinates, having axial symmetry and leading to exact separability of the Hamilton-Jacobi equation for the motion of a satellite. It contains three adjustable constants, $b_{0}, b_{1}$, and $c$, which may be so chosen that the expansion of $V_{L}$ in spherical harmonics is

$$
\begin{aligned}
V_{L}=-\mu r^{-1}[1 & -J_{2}(R / r)^{2} P_{2}(\sin \theta)+J_{2}^{2}(R / r)^{4} P_{4}(\sin \theta)-J_{2}^{3}(R / r)^{6} P_{6}(\sin \theta)+\ldots \\
& \left.\quad-J_{1}(R / r) P_{1}(\sin \theta)+J_{1} J_{2}(R / r)^{3} P_{3}(\sin \theta)-J_{1} J_{2}^{2}(R / r)^{5} P_{5}(\sin \theta)+\ldots\right],
\end{aligned}
$$

where $J_{2}$ is given by (2.1) and where $J_{1}$ is the same as the $J_{1}$ in (1), viz,

$$
J_{1}=-\delta / R \text {. }
$$

$\delta$ being the northerly displacement of the center of mass $C$ from $O$.

Comparison of (4) with (1) shows that the fit is exact for the zeroth harmonic, $-\mu r^{-1}$, and for the first and second harmonics. It would be exact through all harmonics if the planetary distribution of mass happened to be such that

$$
\begin{gathered}
J_{4}=-J_{2}^{2}, \quad J_{6}=J_{2}^{3}, \ldots, \quad J_{2 n}=(-1)^{n+1} J_{n}^{2}, \\
J_{3}=-J_{1} J_{2}, \quad J_{5}=J_{1} J_{2}^{2}, \ldots, \quad J_{2 n+1}=(-1)^{n} J_{1} J_{2}^{n} .
\end{gathered}
$$

\begin{tabular}{|c|c|c|c|c|}
\hline \multirow{2}{*}{ Source } & \multicolumn{4}{|c|}{ Harmonic } \\
\hline & (Zeroth) $10^{6}$ & $-10^{6} J_{2}$ & $-10^{6} J_{4}$ & $-10^{6} J_{6}$ \\
\hline Jeffreys $[3]$ & $10^{6}$ & -1090 & 2.45 & \\
\hline $\begin{array}{l}\text { From fit of } b_{0} \text { and } c \text { to Jef- } \\
\text { freys' } J\end{array}$ & $10^{6}$ & -1 & & $-(1.30) 10^{-3}$ \\
\hline King-Hele and Merson [4] & $10^{6}$ & -1080 & 1. $37 \pm 0.23$ & $-0.1 \pm 1.5$ \\
\hline $\begin{array}{l}\text { From fit of } b_{0} \text { and } \mathrm{c} \text { to their } \\
\quad\end{array}$ & $10^{6}$ & -1080 & 1.17 & $-(1.26) 10^{-3}$ \\
\hline
\end{tabular}

To obtain some idea of the adequacy of the potential (4) let us consider only the even harmonics. From (3) and (4) we may construct the following table of their amplitudes, with signs. 
The fit of the zeroth and second harmonics is necessarily exact. If we fit to Jeffreys' $J$, the resulting fourth harmonic has an amplitude amounting to 49 percent of that given by Jeffreys; the resulting error of (4) then amounts to $1.26 \mathrm{ppm}$, corresponding to an error of 1.26 parts in 1100 for the oblateness potential. If we fit to King-Hele and Merson's $J$, the resulting fourth harmonic has an amplitude amounting to $(86 \pm 16)$ percent of their value for the fourth harmonic, so that the agreement is within the observational error of their determination. Even the sixth harmonic as fitted also lies within their estimates, which are, however, very hazy. In any event the resulting error of (4) then amounts to only $0.20 \mathrm{ppm}$, corresponding to an error of only one part in 5500 for the oblateness correction.

The potential function that we find in this paper, leading to Hamilton-Jacobi separability, is thus much closer to the actual potential, at every point of space, than any heretofore used as the starting point of a calculation. If the results of King-Hele and Merson [4] are confirmed, it may be possible to do orbit theory for unretarded satellites very accurately without perturbations of the solution of the Hamilton-Jacobi equation. If not, there is still a good chance of minimizing the use of perturbation theory, by distributing over the zeroth and second harmonics the secular effect of the fraction of the fourth harmonic that is not accounted for. ${ }^{3}$

\section{Reference System}

As a reference system we first choose a right-handed system of rectangular coordinates, with origin at the center $O$, with $O z$ pointing towards the planet's north celestial pole and with $O x$ pointing towards the planet's vernal equinox. The directions of the axes are then fixed in an inertial system. For the computation of satellite orbits a slow precession of the axis of symmetry about the celestial polar axis may be disregarded, if the precession is sufficiently slow and if the angle of the cone of precession amounts only to about $0.1^{\prime \prime}$, as in the case of the earth [3, p. 202]. Then $O z$ may be considered coincident with the axis of symmetry.

The forces per unit mass acting on a satellite are then, relative to such a system and in the absence of drag:

$$
\mathbf{F}_{1}=\text { gravitational field of the planet }=-\nabla V,
$$

$\mathbf{F}_{2}=$ vector sum of the gravitational fields of the sun and of any moons that the planet may have, plus the inertial forces on the satellite produced by acceleration of the center of mass of the planet by the sun and the moons,

$\mathbf{F}_{3}=$ inertial force on the satellite produced by acceleration of the point $O$ in the planet, relative to its center of mass $C,=-\ddot{\boldsymbol{\delta}}$, where $\delta$ is the vector from $C$ to $O$.

If in (1) we disregard all higher harmonics except the second and calculate $-\nabla V$, we find that the ratio of the amplitude of the oblateness force $\mathbf{F}_{0}$ to the total gravitational force is of the order $4.5 J_{2} \approx 1 / 200$. Thus, for the earth

Furthermore [6],

$$
\left|\mathbf{F}_{0}\right|<5 \mathrm{~cm} / \mathrm{sec}^{-2} .
$$

and

$$
\left|\mathbf{F}_{2}\right| \approx 10^{-4} \mathrm{~cm} / \mathrm{sec}^{-2}
$$

$$
|\ddot{\boldsymbol{\delta}}|=\left(2 \pi / T_{p}\right)^{2} \delta \sin \alpha \approx(1.4) 10^{-20} \delta \sec ^{-2}
$$

for a period $\mathrm{T}_{p}$ of precession $\approx 433$ days [3, p. 202] and for an angle $\alpha$ of the cone of precession $\approx 0.1^{\prime \prime}$. Thus

$$
F_{3} / F_{2} \approx(1.4) 10^{-11} \delta \quad(\delta \text { in kilometers }),
$$

so that $F_{3}$ is utterly negligible compared to $F_{2}$, for any conceivable displacement $\delta$ of $O$ from $C$. In turn, $F_{2}$ amounts to less than $1 / 50,000$ of the maximum oblateness force.

3 The author is indebted to Dr, Boris Garfinkel for this suggestion. 
We are thus left with $\mathbf{F}_{1}$ as the only appreciable force in the absence of drag, and we may choose our coordinate origin either at the center of mass $C$ or at any near point $O$ on the polar axis that leaves $J_{2}$ essentially unchanged but simplifies the odd harmonic part of (1).

\section{Oblate Spheroidal Coordinates}

We next introduce the oblate spheroidal coordinates $\xi$, $\eta$, and $\phi$, defined by

$$
\begin{gathered}
x=c\left[\left(\xi^{2}+1\right)\left(1-\eta^{2}\right)\right]^{\frac{1}{2}} \cos \phi \quad(0<\xi<\infty), \\
y=c\left[\left(\xi^{2}+1\right)\left(1-\eta^{2}\right)\right]^{\frac{1}{2}} \sin \phi \quad(-1 \leqq \eta \leqq 1), \\
z=c \xi \eta,
\end{gathered}
$$

where $c$ is a distance to be fixed later. (For many of the following calculations, the book by Morse and Feshbach [7j is a very useful reference.) The surfaces $\xi=$ constant are oblate spheroids; sections perpendicular to the $z$-axis are circles and meridian sections are confocal ellipses of semiaxes $c\left(\xi^{2}+1\right)^{\frac{1}{2}}$ and $c \xi$, the focal separation being $2 c$. The locus of the foci is thus a circle of radius $c$ in the plane $z=0$. The surfaces $\eta=$ constant are one-sheet hyperboloids of revolution, asymptotic to cones with vertices at the origin, and the surfaces with right ascension $\phi=$ constant are planes containing the polar axis. Note that $\eta= \pm 1$ along $O z$ or $-O z$ respectively and that for sufficiently large values of $r=\left(x^{2}+y^{2}+z^{2}\right)^{\frac{1}{2}}$ the coordinates $\xi$ and $\eta$ behave like $\xi \approx r / c$ and $\eta \approx \sin \theta$, where $\theta$ is the declination [7, p. 1292]. The plane $z=0$ consists of the regions $\eta=0$ and $\xi=0$, outside and inside the focal circle, respectively.

The element of arc $d s$ is given by

$$
d s^{2}=h_{1}^{2} d \xi^{2}+h_{2}^{2} d \eta^{2}+h_{3}^{2} d \phi^{2},
$$

where [7, p. 1292]

$$
\begin{aligned}
& h_{1}=c\left(\xi^{2}+\eta^{2}\right)^{\frac{1}{2}}\left(\xi^{2}+1\right)^{-\frac{1}{2}} \\
& h_{2}=c\left(\xi^{2}+\eta^{2}\right)^{\frac{1}{2}}\left(1-\eta^{2}\right)^{-\frac{1}{2}} \\
& h_{3}=c\left(\xi^{2}+1\right)^{\frac{1}{2}}\left(1-\eta^{2}\right)^{\frac{1}{2}} .
\end{aligned}
$$

\section{Hamilton-Jacobi Equation}

Henceforth all quantities proportional to the mass of the satellite are taken per unit mass of the latter. $\mathrm{By}(9)$ the kinetic energy of the satellite is then given by

$$
T=\frac{1}{2}\left(h_{1}^{2} \dot{\xi}^{2}+h_{2}^{2} \dot{\eta}^{2}+h_{3}^{2} \dot{\phi}^{2}\right),
$$

so that

$$
T=\frac{1}{2}\left(h_{1}^{-2} p_{\xi}^{2}+h_{2}^{-2} p_{\eta}^{2}+h_{3}^{-2} p_{\phi}^{2}\right),
$$

where the generalized momenta are

$$
\begin{aligned}
& p_{\xi}=h_{1}^{2} \dot{\xi}=\partial S / \partial \xi \\
& p_{\eta}=h_{2}^{2} \dot{\eta}=\partial S / \partial \eta \\
& p_{\phi}=h_{3}^{2} \dot{\phi}=\partial S / \partial \phi,
\end{aligned}
$$

$S(\xi, \eta, \phi)$ being the action function. 
For the case of axial symmetry $V=V(\xi, \eta)$ and the Hamiltonian $H$ becomes

$$
H=\left(2 c^{2}\right)^{-1}\left[\frac{\xi^{2}+1}{\xi^{2}+\eta^{2}} p_{\xi}^{2}+\frac{1-\eta^{2}}{\xi^{2}+\eta^{2}} p_{\eta}^{2}+\frac{p_{\phi}^{2}}{\left(\xi^{2}+1\right)\left(1-\eta^{2}\right)}\right]+V(\xi, \eta) .
$$

The Hamilton-Jacobi equation is then

$$
\left(\xi^{2}+1\right)(\partial S / \partial \xi)^{2}+\left(1-\eta^{2}\right)(\partial S / \partial \eta)^{2}+\frac{\xi^{2}+\eta^{2}}{\left(\xi^{2}+1\right)\left(1-\eta^{2}\right)}(\partial S / \partial \phi)^{2}+2 c^{2}\left(\xi^{2}+\eta^{2}\right) V(\xi, \eta)=2 c^{2}\left(\xi^{2}+\eta^{2}\right) \alpha_{1},
$$

where $\alpha_{1}$ is the constant energy.

To try to separate variables in (15), we express $S$ as

$$
S=S_{1}(\xi)+S_{2}(\eta)+S_{3}(\phi),
$$

whereupon (15) becomes

Here

$$
\left(\xi^{2}+1\right) S_{1}^{\prime 2}+\left(1-\eta^{2}\right) S_{2}^{\prime 2}+\left(\frac{1}{1-\eta^{2}}-\frac{1}{\xi^{2}+1}\right) S_{3}^{\prime 2}+2 c^{2}\left(\xi^{2}+\eta^{2}\right) V(\xi, \eta)=2 c^{2}\left(\xi^{2}+\eta^{2}\right) \alpha_{1} .
$$

$$
S_{1}^{\prime}=d S_{1} / d \xi, \quad S_{2}^{\prime}=d S /_{2} d \eta \quad S_{3}^{\prime}=d S_{3} / d \phi .
$$

From (17) it follows that $S_{3}^{\prime 2}$ is expressible as a function of $\xi$ and $\eta$ alone, so that

$$
S_{3}^{\prime}=\alpha_{3}=\text { constant. }
$$

Further inspection of (17) shows that the variables $\xi$ and $\eta$ can be separated if and only if the potential $V(\xi, \eta)$ has the form

$$
V(\xi, \eta)=\left(\xi^{2}+\eta^{2}\right)^{-1}[f(\xi)+g(\eta)] .
$$

The question then arises: Is it possible to find a solution of Laplace's equation, of the form (20), that will adequately represent the known facts about the earth's potential? As indicated in the introduction, the answer is essentially yes. We accordingly devote the next section to finding all the possible solutions of Laplace's equation that have this form.

\section{Solutions of Laplace's Equation That Lead to Hamilton-Jacobi Separability} $1292]$

For axial symmetry, Laplace's equation in oblate spheroidal coordinates becomes [7, p.

$$
\frac{\partial}{\partial \xi}\left[\left(\xi^{2}+1\right) \frac{\partial V}{\partial \xi}\right]+\frac{\partial}{\partial \eta}\left[\left(1-\eta^{2}\right) \frac{\partial V}{\partial \eta}\right]=0 .
$$

Insertion of (20) into (21) then shows that the functions $f(\xi)$ and $g(\eta)$ must satisfy the equation $\xi^{2}\left(\xi^{2}+1\right) f^{\prime \prime}(\xi)-2 \xi\left(\xi^{2}+2\right) f^{\prime}(\xi)+2\left(\xi^{2}+2\right) f(\xi)+\eta^{2}\left(1-\eta^{2}\right) g^{\prime \prime}(\eta)+2 \eta\left(\eta^{2}-2\right) g^{\prime}(\eta)-2\left(\eta^{2}-2\right) g(\eta)$

where

$$
+M(\xi, \eta)=0 \text {, }
$$

$$
\begin{aligned}
M(\xi, \eta) & \equiv \eta^{2} F(\xi)-\xi^{2} G(\eta) \\
F(\xi) & \equiv\left(\xi^{2}+1\right) f^{\prime \prime}(\xi)+2 \xi f^{\prime}(\xi)-2 f(\xi) \\
G(\eta) & \equiv\left(\eta^{2}-1\right) g^{\prime \prime}(\eta)+2 \eta g^{\prime}(\eta)-2 g(\eta) .
\end{aligned}
$$

By (22) a solution of (21) of the form (20) can exist only if $M(\xi, \eta)$ is separable. Now the neces- 
sary and sufficient conditions that $M(\xi, \eta)$ be separable are that $F(\xi)$ and $G(\eta)$ have the forms

$$
\begin{gathered}
F(\xi)=4 B+4 C \xi^{2} \\
G(\eta)=-4 A+4 C \eta^{2},
\end{gathered}
$$

where $A, B$, and $C$ are constants and where the signs and the factor 4 are chosen with a view to later convenience. The conditions are obviously sufficient; to show that they are also necessary, note that if $M(\xi, \eta)$ is separable, there must exist functions $\phi(\xi)$ and $\psi(\eta)$ such that

$$
\eta^{2} F(\xi)-\xi^{2} G(\eta)=\psi(\eta)-\phi(\xi) .
$$

On first placing $\eta=0$ in $(26)$ and then $\xi=0$, we find respectively

$$
\begin{aligned}
& \phi(\xi)=\psi(0)+G(0) \xi^{2} \\
& \psi(\eta)=\phi(0)+F(0) \eta^{2} .
\end{aligned}
$$

Then, on inserting the expressions (27) into (26), we find

$$
\eta^{2}[F(\xi)-F(0)]-\xi^{2}[G(\eta)-G(0)]=\phi(0)-\psi(0) .
$$

Placing either $\xi=0$ or $\eta=0$ in (28) now shows that $\phi(0)=\psi(0)$, so that

$$
\xi^{-2}[F(\xi)-F(0)]=\xi^{-2}[G(\eta)-G(0)]=4 C,
$$

where $C$ is a constant, by a familiar argument. Thus

$$
\begin{aligned}
& F(\xi)=F(0)+4 C \xi^{2} \\
& G(\eta)=G(0)+4 C \eta^{2},
\end{aligned}
$$

which have the general forms indicated in (25), so that the latter are also necessary.

Comparison of (24.1) and (25.1) and of (24.2) and (25.2) shows that the functions $f(\xi)$ and $g(\eta)$ must satisfy

$$
\begin{gathered}
\left(\xi^{2}+1\right) f^{\prime \prime}+2 \xi f^{\prime}-2 f-4 C \xi^{2}-4 B=0 \\
\left(\eta^{2}-1\right) g^{\prime \prime}+2 \eta g^{\prime}-2 g-4 C \eta^{2}+4 A=0 .
\end{gathered}
$$

By (23) and (25) it now follows that

$$
M(\xi, \eta)=4 A \xi^{2}+4 B \eta^{2} .
$$

Insertion of (32) into (22) then shows that the functions $f(\xi)$ and $g(\eta)$ must also satisfy

$$
\begin{aligned}
& \xi^{2}\left(\xi^{2}+1\right) f^{\prime \prime}(\xi)-2 \xi\left(\xi^{2}+2\right) f^{\prime}(\xi)+2\left(\xi^{2}+2\right) f(\xi)+4 \mathrm{~A} \xi^{2}= \\
& \quad \eta^{2}\left(\eta^{2}-1\right) g^{\prime \prime}(\eta)-2 \eta\left(\eta^{2}-2\right) g^{\prime}(\eta)+2\left(\eta^{2}-2\right) g(\eta)-4 B \eta^{2} .
\end{aligned}
$$

The usual argument about separability then shows that each side of (33) is equal to a constant $4 K$. Thus $f(\xi)$ and $g(\eta)$ must also satisfy

$$
\begin{aligned}
& \xi^{2}\left(\xi^{2}+1\right) f^{\prime \prime}-2 \xi\left(\xi^{2}+2\right) f^{\prime}+2\left(\xi^{2}+2\right) f+4 A \xi^{2}-4 K=0 \\
& \eta^{2}\left(\eta^{2}-1\right) g^{\prime \prime}-2 \eta\left(\eta^{2}-2\right) g^{\prime}+2\left(\eta^{2}-2\right) g-4 B \eta^{2}-4 K=0 .
\end{aligned}
$$


We may avoid solving second-order differential equations, as follows: To eliminate $f^{\prime \prime}$, multiply (31.1) by $\xi^{2}$ and subtract the resulting equation from (34.1). To eliminate $g^{\prime \prime}$, multiply (31.2) by $\eta^{2}$ and subtract the resulting equation from (34.2). The resulting first-order equations are the linear ones

$$
\begin{gathered}
\xi f^{\prime}-f=\left(\xi^{2}+1\right)^{-1}\left[C \xi^{4}+(A+B) \xi^{2}-K\right] \\
\eta g^{\prime}-g=\left(\eta^{2}-1\right)^{-1}\left[C \eta^{4}-(A+B) \eta^{2}-K\right] .
\end{gathered}
$$

Their most general solutions are

$$
\begin{gathered}
f(\xi)=K+b_{0} \xi+C \xi^{2}+(A+B-C+K) \xi \tan ^{-1} \xi \\
g(\eta)=-K-b_{1} \eta+C \eta^{2}+\frac{1}{2}(A+B-C+K) \eta \ln \frac{1+\eta}{1-\eta}
\end{gathered}
$$

where $b_{0}$ and $b_{1}$ are constants of integration. Insertion of (36.1) into (31.1) now shows that

$$
B=A
$$

Then, with $B=A$, insertion of (36.2) into (31.2) simply yields a check.

Thus

$$
\begin{gathered}
f(\xi)=K+b_{0} \xi+C \xi^{2}+(2 A-C+K) \xi \tan ^{-1} \xi \\
g(\eta)=-K-b_{1} \eta+C \eta^{2}+\frac{1}{2}(2 A-C+K) \eta \ln \frac{1+\eta}{1-\eta}
\end{gathered}
$$

It is easy to verify that these solutions for $f(\xi)$ and $g(\eta)$ satisfy both sets of second-order equations, viz, (31) and (34), as they should. Moreover, since $f(\xi)$ and $g(\eta)$ must satisfy (35) and since the expressions (36) are the most general solutions of (35), it follows that there are no other solutions for $f(\xi)$ and $g(\eta)$.

From (38) it now follows that all axially symmetric solutions of Laplace's equation that have the form $(20)$ in oblate spheroidal coordinates are expressible as

$$
V(\xi, \eta)=C+\left(\xi^{2}+\eta^{2}\right)^{-1}\left[b_{0} \xi-b_{1} \eta+b_{2}\left(2 \xi \tan ^{-1} \xi+\eta \ln \frac{1+\eta}{1-\eta}\right)\right]
$$

where we have replaced $\frac{1}{2}(2 A-C+K)$ by $b_{2}$. The Hamilton-Jacobi equation is separable, when the potential is a solution of Laplace's equation, if and only if the potential has this form in oblate spheroidal coordinates.

If as usual we take $V$ to be zero at infinity, the constant $C$ vanishes. We are thus left with three explicit adjustable constants, $b_{0}, b_{1}$, and $b_{2}$. It is at once clear, however, that $b_{2}$ must vanish if the solution is to represent the potential outside a planet, for the logarithmic term has a singularity everywhere on the polar axis, for which $\eta= \pm 1$.

We thus finally arrive at a solution of Laplace's equation which makes the HamiltonJacobi equation separable and which contains two explicit adjustable constants, $b_{0}$ and $b_{1}$, and one implicit adjustable constant $c$, the latter appearing in the equations of transformation from oblate spheroidal to rectangular coordinates. This solution is

$$
V=\left(\xi^{2}+\eta^{2}\right)^{-1}\left(b_{0} \xi-b_{1} \eta\right)=b_{0} \operatorname{Re}(\xi+j \eta)^{-1}+b_{1} \operatorname{Im}(\xi+j \eta)^{-1}
$$

The reader may readily verify that $(\xi+j \eta)^{-1}$ is a solution of the Laplace equation (21) and thus that (40) is a solution. (We here use $j$ instead of $i$ for the imaginary unit, saving $i$ for use in later papers as orbital inclination.) 


\section{Expansion of the Potential in Spherical Harmonics}

From (8) we obtain

$$
\begin{aligned}
\xi^{2}-\eta^{2} & =r^{2} / c^{2}-1, \\
\xi \eta & =s r / c,
\end{aligned}
$$

where $r$ is the distance from the origin to the satellite and where

$$
s=\sin \theta,
$$

$\theta$ being the declination of the satellite. Then

where

$$
\begin{aligned}
(\xi+j \eta)^{2} & =\xi^{2}-\eta^{2}+2 j \xi \eta=r^{2} c^{-2}-1+2 j s r c^{-1} \\
& =r^{2} c^{-2}\left(1-2 h s+h^{2}\right)
\end{aligned}
$$

$$
h \equiv-j c r^{-1} .
$$

Then

$$
(\xi+j \eta)^{-1}=c r^{-1}\left(1-2 h s+h^{2}\right)^{-\frac{1}{2}} .
$$

Since we shall choose $c<<r$, it follows that $|h|<1$ and $\left(1-2 h s+h^{2}\right)^{-\frac{1}{2}}$ is then the generating function for the Legendre polynomials $P_{n}(s)$. Thus

$$
(\xi+j \eta)^{-1}=c r^{-1} \sum_{n=0}^{\infty} h^{n} P_{n}(s)=c r^{-1} \sum_{n=0}^{\infty}(-j)^{n} c^{n} r^{-n} P_{n}(s),
$$

from which there follow the relations

$$
\begin{aligned}
& \left(\xi^{2}+\eta^{2}\right)^{-1} \xi=\operatorname{Re}(\xi+j \eta)^{-1}=c r^{-1}\left[1-c^{2} r^{-2} P_{2}(\sin \theta)+c^{4} r^{-4} P_{4}(\sin \theta)-\ldots . .\right] \\
& \left(\xi^{2}+\eta^{2}\right)^{-1} \eta=-\operatorname{Im}(\xi+j \eta)^{-1}=c r^{-1}\left[c r^{-1} P_{1}(\sin \theta)-c^{3} r^{-3} P_{3}(\sin \theta)+c^{5} r^{-5} P_{5}(\sin \theta)-\ldots .\right.
\end{aligned}
$$

From (40) and (45) it then follows that

$$
V=b_{0} c r^{-1}\left[1-c^{2} r^{-2} P_{\Sigma}(\sin \theta)+c^{4} r^{-4} P_{4}(\sin \theta)-\ldots . . b_{1} c r^{-1}\left[c r^{-1} P_{1}(\sin \theta)-c^{3} r^{-3} P_{3}(\sin \theta)+\ldots . .\right]\right.
$$

\section{Evaluation of the Adjustable Constants}

Comparison of (46) with (1) shows that to fit the zeroth harmonic we must choose

$$
b_{0} c=-\mu,
$$

that to fit the second harmonic we must then choose

$$
c^{2}=R^{2} J_{2},
$$

and that to fit the first harmonic we must choose

$$
b_{1} c^{2}=-\mu R J_{1}=\mu \delta .
$$

That is all the flexibility that (46) permits; the higher harmonics are then all fixed, as indicated in the relations (5), which follow from (46).

These choices lead to the form (4) for the potential, which has been amply discussed in the introduction. For $R=6378.388 \mathrm{~km}$ we find from (47.2) and table 1:

$$
\begin{array}{ll}
c=210.7 \mathrm{~km} & \text { (Jeffreys), } \\
c=209.9 \mathrm{~km} & \text { (King-Hele and Merson). }
\end{array}
$$


Although eqs (8) appear to place this constant $c$ in so central a position in the theory as to make numerical predictions depend strongly on its accuracy, it is not really so. Actually we shall see that a knowledge of the value of $c$ is needed only to find the deviations of the orbit from a conic section.

From (40) and (47) we obtain as our working expression for $V$, in oblate spheroidal coordinates:

$$
V=-\mu c^{-1}\left(\xi^{2}+\eta^{2}\right)^{-1}(\xi+\eta \delta / c) .
$$

\section{Formal Solution of the Hamilton-Jacobi Equation}

Insertion of (19) and (49) into (17) now results in the fully separated equation

$$
\left(\xi^{2}+1\right) S_{1}^{\prime 2}-\left(\xi^{2}+1\right)^{-1} \alpha_{3}^{2}-2 \mu c \xi-2 \alpha_{1} c^{2} \xi^{2}=-\left(1-\eta^{2}\right){S_{2}^{\prime}}^{2}-\left(1-\eta^{2}\right)^{-1} \alpha_{3}^{2}+2 \mu \delta \eta+2 \alpha_{1} c^{2} \eta^{2}=k .
$$

By the usual argument, each side of (50) is equal to a constant $k$.

At this point it is necessary to discuss complete and incomplete orbits. We define a complete orbit as the orbit that would be traversed by the satellite when the time $t$ is allowed to run from $-\infty$ to $+\infty$, on the assumption that the equations defining the orbit hold for all values of $t$. In the cases of a planetary satellite or of a meteor coming in from infinity with its orbit being bent around the planet without hitting it, the actual physical orbit is complete. Any projectile fired from a gun and returning to earth, however, exemplifies an incomplete bound orbit. Any missile which escapes from the earth exemplifies an incomplete unbound orbit, as does any meteor with energy $\alpha_{1}>0$ which hits the earth. Note that the complete orbit may or may not intersect the planet. If it does, we define it as that complete orbit which would be described by the satellite, in the absence of planetary matter, but in a gravitational field corresponding to (49). For $r>c$, this field can be represented by the multipole field (46).

By (50) it follows that

$$
k+\alpha_{3}^{2}=-\left(1-\eta^{2}\right) S_{2}^{\prime 2}-\eta^{2}\left(1-\eta^{2}\right)^{-1} \alpha_{3}^{2}+2 \mu \delta \eta+2 \alpha_{1} c^{2} \eta^{2} .
$$

Thus if the plane $\eta=0$ is reached by the complete orbit

$$
k+\alpha_{3}^{2} \leqq 0 .
$$

In the case where $\delta$ is chosen to be zero, the plane $\eta=0$ is the ordinary equatorial plane through the center of mass of the planet. Thus, if this plane is reached by the complete orbit, (52) is satisfied.

It is clear that (52) is only a necessary condition that the complete orbit reach the plane $\eta=0$. It is difficult to find a corresponding sufficient condition. By analogy with the Kepler problem $\left(r^{-1}\right.$ potential), we may suspect that when $\delta=0$ the equatorial plane will always be reached by the complete orbit of any bound satellite. If $\delta \neq 0$, however, the plane $\eta=0$ may not be reached if the orbit is very close to the equator. This latter circumstance is illustrated in figure 1.

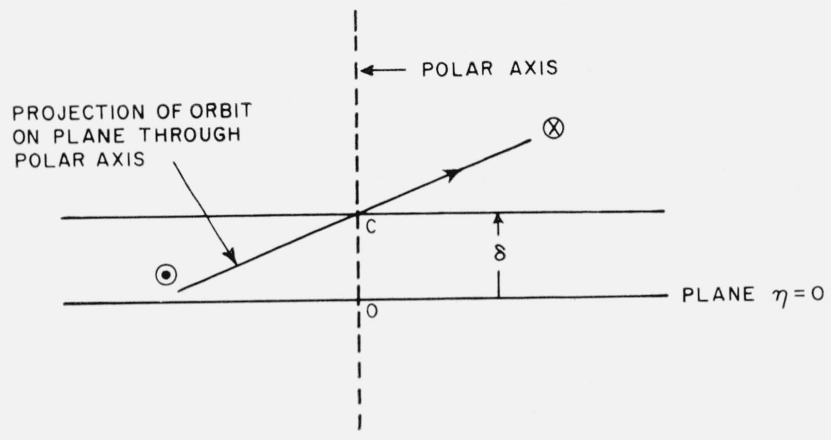


The difficulties in finding a condition sufficient for $\eta=0$ to be reached are illustrated by the existence of cases where the complete orbit may not reach either the region $\eta=0$ outside the focal disk or the region $\xi=0$ inside it. For example, if a particle is dropped from a point on the polar axis into a matter-free region satisfying (49), so that $\alpha_{3}=0, \eta_{0}= \pm 1, \dot{\eta}_{0}=0$, it follows (with $\delta=0$ ) that the complete orbit is a piece of the polar axis, penetrating neither $\xi=0$ nor $\eta=0$.

In the rest of the paper we exclude the possibility of orbits that lie only on the polar axis, thus ruling out, e.g., the case of a missile fired vertically from either pole. We then consider only those orbits whose completions either reach the plane $\eta=0$ or reach at least one genuine extremum $\eta_{1}$ for which $\dot{\eta}=0, \ddot{\eta} \neq 0$.

From (50) it now follows that

$$
\begin{aligned}
& S_{1}^{\prime}(\xi)= \pm\left(\xi^{2}+1\right)^{-1}\left[\alpha_{3}^{2}+\left(k+2 \mu c \xi+2 \alpha_{1} c^{2} \xi^{2}\right)\left(\xi^{2}+1\right)\right]^{\frac{1}{2}} \\
& S_{2}^{\prime}(\eta)= \pm\left(1-\eta^{2}\right)^{-1}\left[-\alpha_{3}^{2}+\left(-k+2 \mu \delta \eta+2 \alpha_{1} c^{2} \eta^{2}\right)\left(1-\eta^{2}\right)\right]^{\frac{1}{2}},
\end{aligned}
$$

where by (13), (16), and (18) the upper sign is to be used whenever the corresponding coordinate is increasing and the lower sign whenever it is decreasing. Also

$$
S_{3}^{\prime}(\phi)=\alpha_{3} .
$$

From (53) and (19) it then follows that

$$
\begin{aligned}
S=\alpha_{3} \phi \pm \int_{\xi_{1}}^{\xi}\left(\xi^{2}+1\right)^{-1}\left[\alpha_{3}^{2}+(k+\right. & \left.\left.2 \mu c \xi+2 \alpha_{1} c^{2} \xi^{2}\right)\left(\xi^{2}+1\right)\right]^{\frac{1}{2}} d \xi \\
& \pm \int_{\eta_{1}}^{\eta}\left(1-\eta^{2}\right)^{-1}\left[-\alpha_{3}^{2}+\left(-k+2 \mu \delta \eta+2 \alpha_{1} c^{2} \eta^{2}\right)\left(1-\eta^{2}\right)\right]^{\frac{1}{2}} d \eta+\text { const. }
\end{aligned}
$$

Before defining the lower limits $\xi_{1}$ and $\eta_{1}$, it is well to note that eq (54) is somewhat deceptive in appearance, because of the presence of the "small" distance $c$ in terms that are clearly very important. Let us now remove this difficulty, as follows. Replace the variable $\xi$ by the variable $\rho$, where

By (41) it then follows that

$$
\begin{gathered}
\rho \equiv c \xi . \\
\rho^{2}=r^{2}-\left(1-\eta^{2}\right) c^{2} \\
\rho \eta=r \sin \theta \\
\xi^{2}+1=\left(\rho^{2}+c^{2}\right) c^{-2},
\end{gathered}
$$

so that $\rho \approx r$ on the physically realizable part of any complete orbit. On substituting (55) into (54) and dropping the constant term, we obtain

$$
\begin{aligned}
S=\alpha_{3} \phi \pm \int_{\rho_{1}}^{\rho}\left(\rho^{2}+c^{2}\right)^{-1}\left[c^{2} \alpha_{3}^{2}+(k+2 \mu \rho\right. & \left.\left.+2 \alpha_{1} \rho^{2}\right)\left(\rho^{2}+c^{2}\right)\right]^{\frac{1}{2}} d \rho \\
& \pm \int_{\eta_{1}}^{\eta}\left(1-\eta^{2}\right)^{-1}\left[-\alpha_{3}^{2}+\left(-k+2 \mu \delta \eta+2 \alpha_{1} c^{2} \eta^{2}\right)\left(1-\eta^{2}\right)\right]^{\frac{1}{2}} d \eta,
\end{aligned}
$$

where

$$
\rho_{1}=c \xi_{1}
$$

If the complete orbit intersects the plane $\eta=0$, we choose the lower limit $\eta_{1}$ to be zero, corresponding to $\theta=0$, in accord with the usual canonical treatment [8] of a bound orbit for the case $V=-\mu r^{-1}$; this appears to be a good choice for an unbound orbit also. If the complete orbit does not intersect the equatorial plane, we choose $\eta_{1}$ as an extremum of $\eta$ on the complete orbit. It will be a zero of the $\eta$-integrand satisfying $\eta \geqq-1$. 
To choose a value for $\rho_{1}$, we reason as follows. The $\rho$-integrand of (57) has the factor

where

$$
F(\rho)=c^{2} \alpha_{3}^{2}+\left(\rho^{2}+c^{2}\right) f(\rho),
$$

$$
f(\rho)=2 \alpha_{1} \rho^{2}+2 \mu \rho+k .
$$

The appropriate real zeros of $F(\rho)$ will be almost equal to those of $f(\rho)$, which always has two in any physically realizable case. If $\alpha_{1} \geqq 0$, one zero of $f(\rho)$ will be positive and the other negative (approaching $-\infty$ as $\alpha_{1}$ approaches zero). If $\alpha_{1}<0$, both zeros of $f(\rho)$ will be positive. Any other real zeros of $F(\rho)$ will occur for inappropriate values of $\rho$, of the order $c$. Thus for unbound orbits, we choose $\rho_{1}$ to be the largest positive zero of $F(\rho)$, this being the only one accessible to complete unbound orbits. For bound orbits, we choose $\rho_{1}$ to be the next to the largest positive zero of $F(\rho)$, equal approximately to the smaller zero of $f(\rho)$. In either case $\rho_{1}$ is then the minimum value of $\rho$ dynamically accessible to the complete orbit; the corresponding spheroid may or may not lie within the earth. (Any value of $\rho$ less than $\rho_{1}$ as just defined is dynamically inaccessible to the complete orbit in the field $V(\xi, \eta)$, even if the planetary matter could be removed without change in $V(\xi, \eta)$ : to reach such values the orbit would have to pass through regions in which $F(\rho)$ is negative and $p_{\xi}$ complex.) Of course $\rho_{1}>R$ and thus outside the earth for any ordinary satellite in a bound orbit.

Whenever the plane $\eta=0$ is intersected by the complete orbit, we have seen that $k \leqq-\alpha_{3}^{2}$, so that we can then introduce a real $\alpha_{2}$ such that

where we can assume

$$
k=-\alpha_{2}^{2},
$$

without loss of generality. Then

$$
\alpha_{2}>0
$$

$$
\alpha_{2}^{2} \geqq \alpha_{3}^{2} .
$$

If we put $\delta=0$ and $c=0$ in (57) and then use $\rho=r, \eta=\sin \theta$, which would then follow from (56), and also use (59.1), we find that (57) reduces [8] to the correct expression for the action function for the case $V=-\mu r^{-1}$. Thus the terms in (57) that contain $c^{2}$ or $\delta$ give rise to deviations from a conic-sectional orbit.

From (13.3) and (19) it follows that the constant $\alpha_{3}$ in (57) is the z-component of angular momentum. The constant $k$ has no name, but is related to the magnitude of the total angular momentum, which is, however, not conserved in the non-central field produced by oblateness. To see the connection, note that whenever $k \leqq-\alpha_{3}{ }^{2}$, we can write (59.1) and (59.3). Then when $\delta$ and $c^{2}$ are both allowed to approach zero in (57), $\alpha_{2}$ approaches the magnitude of the total angular momentum, which is then conserved. (This result follows most easily from the fact already mentioned, viz, that (57) then reduces to the correct action function for the case $V=-\mu r^{-1}$.)

\section{Kinetic Equations of Motion}

Implicit equations for $\rho, \eta$, and $\phi$ as functions of $t$ are now given by

$$
\begin{gathered}
\partial S / \partial \alpha_{1}=t+\beta_{1} \\
\partial S / \partial k=\beta_{2}^{\prime} \\
\partial S / \partial \alpha_{3}=\beta_{3}
\end{gathered}
$$

where $t$ is the time and the $\beta$ 's are constants. Since the lower limit $\rho_{1}$ in (57) is a function of $\alpha_{1}, k$, and $\alpha_{3}$, the derivative of the $\rho$-integral with respect to any of them is the sum of an integral and a term like $\mp \mathrm{I}_{\rho}\left(\rho_{1}\right) \partial \rho_{1} / \partial \alpha_{1}$. Here $\mathrm{I}_{\rho}(\rho)$ is the $\rho$-integrand, which vanishes for $\rho_{1}$, so that this second term vanishes. If the complete orbit intersects the plane $\eta=0$, so that $\eta_{1}$ can be taken to be zero, on second term arises in a derivative of the $\eta$-integral; if not, $\eta_{1}$ is to be 
taken as an extremum value of $\eta$ on the complete orbit and the second term again vanishes, since $\eta_{1}$ is then a zero of the $\eta$-integrand.

The kinetic equations are thus

$$
\begin{gathered}
\pm \int_{\rho_{1}}^{\rho} \rho^{2}\left[c^{2} \alpha_{3}^{2}+\left(k+2 \mu \rho+2 \alpha_{1} \rho^{2}\right)\left(\rho^{2}+c^{2}\right)\right]^{-\frac{1}{2}} d \rho \pm c^{2} \int_{\eta_{1}}^{\eta} \eta^{2}\left[-\alpha_{3}^{2}+\left(-k+2 \mu \delta \eta+2 \alpha_{1} c^{2} \eta^{2}\right)\left(1-\eta^{2}\right)\right]^{-\frac{1}{2}} d \eta=t+\beta_{1}, \\
\quad \pm \frac{1}{2} \int_{\rho_{1}}^{\rho}\left[c^{2} \alpha_{3}^{2}+\left(k+2 \mu \rho+2 \alpha_{1} \rho^{2}\right)\left(\rho^{2}+c^{2}\right)\right]^{-\frac{1}{2}} d \rho \mp \frac{1}{2} \int_{\eta_{1}}^{\eta}\left[-\alpha_{3}^{2}+\left(-k+2 \mu \delta \eta+2 \alpha_{1} c^{2} \eta^{2}\right)\left(1-\eta^{2}\right)\right]^{-\frac{1}{2}} d \eta=\beta_{2}^{\prime},
\end{gathered}
$$

$$
\begin{aligned}
& \phi \pm c^{2} \alpha_{3} \int_{\rho^{1}}^{\rho}\left(\rho^{2}+c^{2}\right)^{-1}\left[c^{2} \alpha_{3}^{2}+\left(k+2 \mu \rho+2 \alpha_{1} \rho^{2}\right)\left(\rho^{2}+c^{2}\right)\right]^{-\frac{1}{2}} d \rho \\
& \mp \alpha_{3} \int_{\eta_{1}}^{\eta}\left(1-\eta^{2}\right)^{-1}\left[-\alpha_{3}^{2}+\left(-k+2 \mu \delta \eta+2 \alpha_{1} c^{2} \eta^{2}\right)\left(1-\eta^{2}\right)\right]^{-\frac{1}{2}} d \eta=\beta_{3} .
\end{aligned}
$$

If we restrict considerations to those cases where the plane $\eta=0$ is intersected by the complete orbit, then $k \leqq \alpha_{3}^{2}$. We can then substitute $k=-\alpha_{2}^{2} \leqq-\alpha_{3}^{2}$ in (57) and replace (60.2) by

$$
\partial S / \partial \alpha_{2}=\beta_{2} .
$$

From (57), (60.1), (60.3), and (62) there then follow the kinetic equations

$$
\begin{aligned}
& \pm \int_{\rho_{1}}^{\rho} \rho^{2}\left[c^{2} \alpha_{3}^{2}+\left(-\alpha_{2}^{2}+2 \mu \rho+2 \alpha_{1} \rho^{2}\right)\left(\rho^{2}+c^{2}\right)\right]^{-\frac{1}{2}} d \rho \pm c^{2} \int_{0}^{\eta} \eta^{2}\left[-\alpha_{3}^{2}+\left(\alpha_{2}^{2}+2 \mu \delta \eta\right.\right. \\
& \left.\left.+2 \alpha_{1} c^{2} \eta^{2}\right)\left(1-\eta^{2}\right)\right]^{-\frac{1}{2}} d \eta=t+\beta_{1}, \\
& \mp \alpha_{2} \int_{\rho_{1}}^{\rho}\left[c^{2} \alpha_{3}^{2}+\left(-\alpha_{2}^{2}+2 \mu \rho+2 \alpha_{1} \rho^{2}\right)\left(\rho^{2}+c^{2}\right)\right]^{-\frac{1}{2}} d \rho \pm \alpha_{2} \int_{0}^{\eta}\left[-\alpha_{3}^{2}+\left(\alpha_{2}^{2}+2 \mu \delta \eta+2 \alpha_{1} c^{2} \eta^{2}\right)\left(1-\eta^{2}\right)\right]^{-\frac{1}{2}} d \eta=\beta_{2} \text {, }
\end{aligned}
$$$$
\phi \pm c^{2} \alpha_{3} \int_{\rho_{1}}^{\rho}\left(\rho^{2}+c^{2}\right)^{-1}\left[c^{2} \alpha_{3}^{2}+\left(-\alpha_{2}^{2}+2 \mu \rho+2 \alpha_{1} \rho^{2}\right)\left(\rho^{2}+c^{2}\right)\right]^{-\frac{1}{2}} d \rho
$$$$
\mp \alpha_{3} \int_{0}^{\eta}\left(1-\eta^{2}\right)^{-1}\left[-\alpha_{3}^{2}+\left(\alpha_{2}^{2}+2 \mu \delta \eta+2 \alpha_{1} c^{2} \eta^{2}\right)\left(1-\eta^{2}\right)\right]^{-\frac{1}{2}} d \eta=\beta_{3} .
$$

Future papers will be devoted to evaluating the integrals in these equations, solving them simultaneously for $\rho, \eta$, and $\phi$ as functions of time, and deriving the astronomical results that they imply, both for bound orbits and unbound orbits.

\section{References}

[1] J. P. Vinti, Phys. Rev. Letters 3, No. 1 (July 1959).

[2] R. H. Merson and D. G. King-Hele, Nature 182, 640 (Sept. 1958).

[3] H. Jeffreys, The earth, 3d ed (Cambridge Univ. Press, Cambridge, England, 1952). See p. 127 at bottom; p. 129 , eq (5); and p. 132, eq (13).

[4] D. G. King-Hele and R. H. Merson, Nature 183, 881 (March 1959).

[5] J. A. O'Keefe, A. Eckels, and R. K. Squires, Science 129, 565 (Feb. 1959).

[6] J. A. O'Keefe, Astron. J. 62, 265 (1957).

[7] P. M. Morse and H. Feshbach, Methods of theoretical physics, vols. I and II (McGraw-Hill Book Co., Inc., New York, N.Y., 1953).

[8] W. M. Smart, Celestial mechanies, p. 143 (Longmans, Green \& Co., New York, N.Y., 1953).

Washington, D.C.

(Paper 63B2-12) 